\title{
Purpura Fulminans with Peripheral Gangrene in Severe Falciparum Malaria: A Case Series
}

\author{
A Fowotade $^{1}$, RE Oladokun ${ }^{2}$, OE Belloº ${ }^{2}$ EO Aigbovo ${ }^{1}$ \\ ${ }^{1}$ Department of Medical Microbiology and Parasitology, College of Medicine, University of Ibadan and University College Hospital, \\ Ibadan, Nigeria. ${ }^{2}$ Department of Paediatrics, College of Medicine, University of Ibadan, Nigeria.
}

\section{Corresponding Author: \\ Dr. Adeola Fowotade \\ Email: temilabike@gmail.com}

This is an Open Access article distributed under the terms of the Creative Commons Attribution License (creativecommons.org/ licenses/by/3.0).

Received Accepted Published

November 30, 2017

December 18, 2017

January 30, 2018

\begin{abstract}
Background: Purpura fulminans is an acute fatal illness consisting of septicaemia, shock, and disseminated intravascular coagulation, often manifesting with gangrene of the distal extremities and necrosis of skin. Purpura fulminans associated with severe malaria has rarely been reported among children. Case Report: Two cases of purpura fulminans with disseminated intravascular coagulation probably due to severe falciparum malaria, are presented from a tertiary health facility in Nigeria. Both cases were males, and were infants aged 6 and 7 months respectively. They presented with fever, seizures, loss of consciousness and features of shock and had associated peripheral gangrene which developed hours into admission. Investigations showed P. falciparum hyper-parasitemia and evidence of disseminated intravascular coagulation. No focus for sepsis was identified and there were no bacterial isolates from blood culture. The second child survived but was left with amputated digits and audiovisual deficits. Conclusion: The cases presented demonstrate that purpura fulminans can occur in children with severe malaria even in P. falciparum endemic settings.
\end{abstract}

Keywords: Child, Falciparum Malaria, Gangrene, Necrosis, Purpura Fulminans.

\section{Introduction}

Purpura fulminans (PF) is a rare life threatening disorder associated with rapidly progressive intravascular thrombosis and hemorrhagic infarction of the skin often resulting in gangrene of the distal extremities and necrosis of the skin. $\mathrm{PF}$ can occur in three main forms; inherited or congenital protein $\mathrm{C}$ abnormalities, idiopathic, or acute infectious disease [1]. PF has been described as a cutaneous marker of disseminated intravascular coagulopathy $[1,2]$. PF is usually associated with many infections, most importantly meningococcal, staphylococcal, and streptococcal infections [3]. In meningococcaemia, PF is one of the most severe presentations and is associated with a mortality of over 20 percent [4]. Other less commonly associated infections include gram-negative agents such as Klebsiella spp. [5,6].
There had been a few published reports of PF associated with malaria especially in adults [7-9]. Malaria remains a major health problem of public health concern, particularly in most parts of Asia and Africa. The WHO African Region carries a disproportionately high share of the global malaria burden. In 2015, the region was home to $90 \%$ of malaria cases and $92 \%$ of malaria deaths. The majority of global malaria deaths (99\%) are due to $P$. falciparum [10].

Malaria transmission in Nigeria is high (hyper-endemic). Furthermore, malaria is one of the three leading causes of deaths among children aged between one month and 59 months [11]. Plasmodium falciparum is the predominant malaria species. Falciparum malaria is associated with complications, including cerebral malaria, severe anaemia, hypoglycaemia and circulatory collapse, and abnormal bleeding [12]. 
It is important that physicians are aware of the complications and management of severe malaria. Infants are among the population groups that are at considerably higher risk of contracting malaria, and developing severe disease. We hereby report on two infants with purpura fulminans with high $P$. falciparum parasitaemia. Both cases highlight a rare cutaneous manifestation of severe malaria and also seek to increase awareness of the fact that purpura fulminans could be associated with malaria in an endemic setting.

\section{Case Reports}

\section{Case 1}

This was a 6 months old male who presented with a two days history of fever and four hours history of difficulty in breathing. The difficulty in breathing gradually worsened with bluish discoloration of the lips and extremities. There were no significant events during pregnancy, birth and neonatal periods. On examination, he was acutely ill, icteric, hypothermic (temperature $35.4^{\circ} \mathrm{C}$ ) and had feeble brachial pulse. The weight was $7.5 \mathrm{~kg}$. The central nervous system examination revealed an unconscious patient with Glasgow Coma Scale (GCS) of 9/15. Both pupils reacted to light. There was normal tone in all the limbs. He was dyspneic as evidenced by grunting respiration, flaring nares and subcostal as well as intercostal recessions. Breath sounds were vesicular with transmitted sounds. Abdominal examination showed hepatosplenomegaly. An assessment of severe malaria was made plus probable septic shock.

Blood film for malaria parasite showed a parasite density of $182,610 / \mu \mathrm{L}$. Twelve hours into admission, he developed focal seizures involving the left upper limbs. His condition further deteriorated. He remained unconscious with circulatory collapse and features of raised intracranial pressure. He was transfused with $20 \mathrm{ml} / \mathrm{kg}$ of whole blood on account of severe anemia (hematocrit at admission was $7 \%$ ). He was transfused with fresh frozen plasma and received multiple whole blood transfusions. Platelet was not available for transfusion. Other management included intravenous artesunate, ceftriaxone and dopamine drip at $5 \mu \mathrm{g} / \mathrm{kg} / \mathrm{min}$. Intravenous phenobarbitone was given on account of seizures. Recurrent episodes of hypoglycaemia were managed with $12.5 \%$ dextrose concentration. Multidisciplinary approach in his care involved surgical and medical units as necessary.

On the second day of admission, he was observed to have bilateral digital gangrene with ecchymosis of the dorsum and medial aspect of the left foot [Fig.1]. Additionally, he developed prolonged bleeding from puncture sites, dark coloured urine and dark effluent from the nasogastric tube. Peripheral blood film showed microcytosis, macrocytosis, moderate hypochromia, few target cells, burr cells, fragmented cells, non-nucleated red blood cells (RBCs), circulating megakaryoblasts [5/100 white blood cells-(WBCs)] and polychromasia. There was also neutrophilia with left shift to myelocyte stage, and severe toxic granulations as well as megathrombocytes. Full blood count (FBC) showed leucocytosis and thrombocytopenia $\left(28,000 / \mathrm{mm}^{3}\right)$. Electrolytes, urea and creatinine $(\mathrm{E} / \mathrm{U} / \mathrm{Cr})$ showed hyperkalaemia $(6.9 \mathrm{mg} / \mathrm{dL})$ and metabolic acidosis. Viral haemorrhagic fever screen for Ebola, Lassa fever, dengue and yellow fever viruses by polymerase chain reaction was negative.

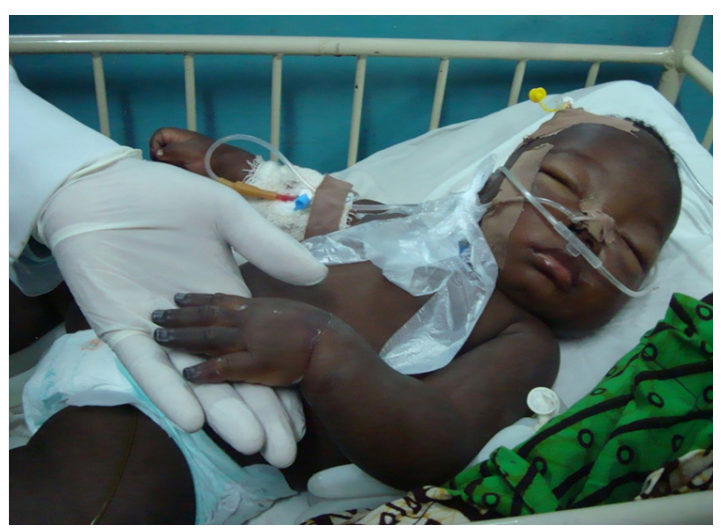

Fig.1: Case 1 with the gangrenous digit shown above the gloved hand. 
HIV test was also negative. The prothrombin time and partial thromboplastin time were prolonged but the fibrin degradation products could not be done. Blood culture carried out on the second day of admission was sterile. The parasite density decreased from 182,610 parasites/ $\mu \mathrm{L}$ on day one to 125 parasites/ $\mu \mathrm{L}$ on day six of admission.

His temperature ranged from $35.4^{\circ} \mathrm{C}$ to $39.6^{\circ} \mathrm{C}$ and urinary output from $0.9 \mathrm{ml} / \mathrm{kg} / \mathrm{hr}$ to $5.4 \mathrm{ml} / \mathrm{kg} / \mathrm{hr}$. He regained full consciousness on the $11^{\text {th }}$ day on admission but remained very ill. On the $14^{\text {th }}$ day of admission, he had worsening dyspnea and suffered a cardiopulmonary arrest and did not respond to resuscitation. Post-mortem lumbar puncture yielded a uniformly bloody cerebrospinal fluid (CSF).

\section{Case 2}

This is a 7 month old male infant who presented with fever and vomiting of two days duration. He developed seizures and loss of consciousness at presentation. There was associated diarrhea. Pregnancy, birth and neonatal history were not adversely eventful. He was up to date with his immunisation. Examination revealed a critically ill infant with a temperature of $39^{\circ} \mathrm{C}$, and severe pallor. The GCS was 12/15. The anterior fontanelle was flat and normotensive. The weight was $6.8 \mathrm{~kg}$. There was reduced tone in all the limbs. Respiratory system examination revealed a dyspneic child with subcostal, intercostal recessions and grunting respiration. The heart rate was 168 beats per minute and he had small volume pulses with a gallop rhythm. Abdominal examination revealed hepatomegaly of $4 \mathrm{~cm}$ and splenomegaly of $6 \mathrm{~cm}$ with hypoactive bowel sound. An assessment of severe malaria (cerebral malaria, severe anaemia and shock) and sepsis was made. Full blood count showed leucocytosis with neutrophil predominance and thrombocytopenia.

The initial treatment included intravenous artesunate and ceftriaxone. About eight hours into admission, the GCS dropped to 5/15 and the digits were noted to have a darkish hue which gradually progressed to gangrene. He also had hyperpigmented patches on the chest wall and purpuric lesions on the lower limbs [Fig.2]. Supportive care included fluid and electrolyte replacement as indicated, intranasal oxygen and intravenous phenobarbitone on account of seizures. The urine output ranged from oliguria to polyuria. He had clinical evidence of raised intracranial pressure (worsening level of unconsciousness and fundoscopy showed blurred optic disc margins). Additionally, flame shaped hemorrhages were seen on the disc. Persistent hypoglycaemia was managed with $12.5 \%$ Dextrose. As a result of the protracted hypotension, septic shock and hypoglycaemia, he was subsequently commenced on intravenous dopamine and hydrocortisone. The Plastic and Orthopaedic surgery teams were involved in the multi-disciplinary management.

Rapid diagnostic (antigen) test (RDT) for malaria was positive and blood film showed hyper-parasitaemia. Parasite density decreased from 288,356 parasites/ $\mu \mathrm{L}$ on day one to 1,314 parasites $/ \mu \mathrm{L}$ on day nine of admission. Other blood tests showed leucocytosis, thrombocyopaenia, metabolic acidosis and features of acute kidney failure. Urinalysis showed: protein $2+$, blood $2+$, $\mathrm{pH} 5$, specific gravity 1.015. Urine microscopy

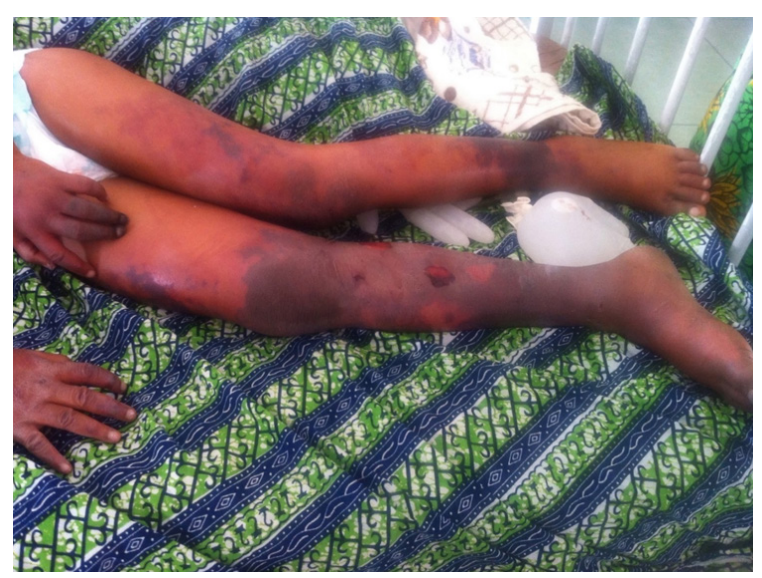

Fig.2: Case 2 with purpuric lesions on the lower limbs. 
and culture were negative. The clotting profile was deranged. HIV test was non-reactive, viral haemorrhagic fever screen was negative. Blood culture could only be carried out on the fifth day of admission and was sterile. CSF analysis was deferred on account of his critical condition.

After one week of intravenous artesunate, with persistence of malaria parasitaemia, clindamycin was added to his treatment. Malaria parasitaemia cleared on the $10^{\text {th }}$ day of admission with marked clinical improvement and regaining of consciousness. The temperature normalised and the progression of the gangrene had arrested. Enteral feeding was gradually reintroduced with re-establishment of breastfeeding. When the level of consciousness improved, artemeter-lumifantrine was added for three days to complete the antimalarial treatment. Lines of demarcation appeared with subsequent auto-amputation of the gangrenous digits. The child was also had residual audiovisual impairment.

\section{Discussion}

The cases of purpura fulminans against an evidence based background of malaria infection in this report illustrate an unusual, yet often fatal manifestation of malaria. Both cases had impaired consciousness and respiratory distress. Most interestingly, they all presented with cutaneous signs of disseminated intravascular coagulation, which rarely develops in severe falciparum malaria. To the best of our knowledge, this is one of the earliest reports of falciparum malaria complicated with $\mathrm{PF}$ in an endemic malaria setting and involving children. Previous studies have shown that 60 to $70 \%$ cases of acute purpura fulminans occur amongst children below two years of age [13]. The two previous cases of PF reported previously in Nigeria involved a two month old and three year-old with symmetric gangrene. Malaria testing was not documented in the reports $[5,6]$.

The previous reports of PF associated falciparum malaria in adults were in a 19 and 44 year old patients in India as well as a 67 year old Japanese woman who contracted malaria while in Africa [9]. PF appears to occur more commonly in infants and younger children. Herein, both cases described were aged less than two years. In the cases presented in our report, the association with malaria is strong especially as both antigen testing and microscopy tests confirmed the disease. A limitation with basing the diagnosis of malaria with presence of parasitaemia is that malaria parasitaemia may be incidental, occurring in the presence of other concurrent disease. However, the level of parasitaemia in both cases was high and showed consistent reduction with treatment over time. The presence of the retinal haemorrhage in the second case also adds evidence to the diagnosis of malaria [14].

Additionally, bacteriological evidence for sepsis was not shown in the two cases as the blood cultures were sterile. Both children had commenced antibiotics prior to the cultures; perhaps this could explain the lack of an isolate. It is difficult to clinically distinguish PF due to bacteria sepsis or P. falciparum. Where the facilities are available, it would have been pertinent to identify the presence or absence of any coexisting pathogen by molecular tests such as DNA PCR. Nevertheless, as PF has commonly been associated with meningococcemia and other bacterial causes, clinical presentation of $\mathrm{PF}$ in the face of the $P$. falciparum parasitaemia should still require empirical treatment with a broad spectrum antibiotic such as ceftriaxone. The use of vasopressor agents such as dopamine in the two patients was indicated as a result of their state of protracted shock. The shock in itself might have also worsened the skin ischemia.

In the firstcase, inspite of antibiotics and antimalarial treatment along with fluid replacements and blood transfusions the illness progressed with a fatal outcome. PF is generally associated with poor outcome as observed in other reports $[2,15,16]$. The second case survived against a lot of odds inspite of the initial protracted shock and multi- 
organ dysfunction with the lack of intensive care. As a result of the multi-organ dysfunction usually associated with PF, the management of the cases of malaria associated PF in the previous reports, consisted of hemo-filtration, plasma exchange and renal dialysis $[8,9]$.

A positive test of generation of thrombin is usually required to diagnose disseminated intravascular coagulopathy (DIC) [17]. Lack of facilities and fund constraints mitigated against assessing levels of the coagulation factors. The bleeding time and clotting time were prolonged as well as thrombocytopenia which indicated disseminated intravascular coagulopathy (DIC). It has been postulated that Shwartzman and Arthus reactions might be responsible for the pathogenesis of purpura fulminans, however the exact mechanisms behind these reactions are poorly understood [18].

The pathogenesis of PF in falciparum malaria is poorly understood. The coagulopathy in falciparum malaria has been linked to multiple factors including high plasma levels of plasminogen activator inhibitor-1 as well as elevated plasma levels of von Willebrand factor (vWF), vWF propeptide, thrombomodulin and endothelial microparticles [19]. Furthermore, acute endothelial cell activation, abnormal circulating ultra large vWF multimers have been demonstrated in severe malaria. Cumulatively these changes might explain the pathogenesis behind the thrombocytopenia and intravascular platelet aggregation. Transfusion of coagulation factor, protein $\mathrm{C}$, protein $\mathrm{S}$, antithrombin III concentrates, have been shown to be beneficial in patients with PF and DIC $[1,13]$.

\section{Conclusion}

Severe malaria may be complicated by purpura fulminans which mimics meningococcemia in its clinical manifestation. Therefore, it is recommended that malaria be considered a differential in infants in malaria endemic settings presenting with purpura fulminans and associated peripheral gangrene.

Contributors: FA, ORE: manuscript writing, patient management; BOE, AEO: manuscript editing, patient management; ORE: critical inputs into the manuscript. ORE will act as guarantor. All authors approved the final version of the manuscript.

Funding: None; Competing interests: None stated.

\section{References}

1. Chalmers E, Cooper P, Forman K. Purpura fulminans: recognition, diagnosis and management. Arch Dis Child. 2011;96:1066-1071.

2. Ghosh SK, Bandyopadhyay D, Dutta A. Purpura fulminans: a cutaneous marker of disseminated intravascular coagulation. West $\mathrm{J}$ Emerg Med. 2009; 10:41.

3. Johansen K, Hansen ST. Symmetrical peripheral gangrene (purpura fulminans) complicating pneumococcal sepsis. Am J Surg.1993;165:642-645.

4. Leclerc F, Binoche A, Dubos F. Meningococcal purpura fulminans in children. Rev Prat. 2004;15:957-962.

5. Olowu WA. Klebsiella-induced purpura fulminans in a Nigerian child: Case report and a review of literature. West Afr J Med. 2002;21:253-256.

6. Umar LW, Ya'uba MS, Olorukooba AA, Abubakar Y, Mohammed AJ, Chom ND. Purpura fulminans with disseminated intravascular coagulopathy and symmetric peripheral gangrene complicating sepsis in an Infant: A Case Report. Ann Med Health Sci Res. 2017;7:69-72.

7. Keri JE, Thomas K, Berman B, Falabella A. Purpura fulminans in a patient with malaria. Eur J Dermatol. 2000;10:617-619.

8. Kato Y, Ohnishi K, Sawada Y, Suenaga M. Purpura fulminans: an unusual manifestation of severe falciparum malaria. Trans R Soc Trop Med Hyg. 2007;101:10451047.

9. Khaira A, Gupta V, Gupta A, Mahajan S, Bhowmik D, Tiwari SC. Purpura fulminans in complicated falciparum malaria. JAPI 2008;56;467-469.

10. World Malaria Report 2016. Geneva: World Health Organization; 2016. Licence:CC BY-NC-SA 3.0 IGO.

11. National Population Commission (Nigeria), Federal Ministry of Health of Nigeria, National Bureau of Statistics (Nigeria), and Institute for International Programs at Johns Hopkins Bloomberg School of Public Health. A verbal/social autopsy study to improve estimates of the causes and determinants of neonatal and child mortality in Nigeria, 2014. Abuja, Nigeria, and Baltimore, Maryland, USA. 2016. Available at: https:// 
www.healthynewbornnetwork.org/hnn-content/uploads/ Nigeria_2014-VASA-Study_final_report.pdf. Accessed on November 30, 2017.

12. WHO. Severe Malaria. WHO. Tropical Medicine and International Health. 2014;19(Suppl. 1):7-131.

13. Darmstadt GL. Acute infectious purpura fulminans: pathogenesis and medical management. Ped Dermatol. 1998;15:169-183.

14. Taylor TE, Molyneux ME. The pathogenesis of paediatric cerebral malaria: eye exams, autopsies and neuro-imaging. Ann N Y Acad Sci. 2015;1342:44-52.

15. Akar M, Demirel FG, Sandal G. Neonatal Purpura fulminans: A report of seven cases. Turkish J Pediatr Dis. 2011;5:95-99.
16. Piparsania S, Rajput N, Singh K. Purpura fulminans in infantile streptococcal septicemia. Surgical Sci. 2011;2:496-498.

17. Calverley DC, Liebman HA. In: Hematology. Basic Principles and Practice, 3rd edition. Hoffman R, Benz EZ, et al. Philadelphia: Churchill Livingstone, Inc. 2000;1983-95.

18. David ZJ, Chu, William BF. Purpura fulminans. The American Journal of Surgery. 1982;143:356-362.

19. Angchaisuksir P. Coagulopathy in malaria. Thrombosis Research. 2014;133:5-9. 